Notes de lecture

\title{
Marie-France Lange et Nolwen Henaff (sous la direction de), « Internationalisation et transformation des systèmes éducatifs du Sud »
}

Revue Tiers-Monde, n 223, juil-sept. 2015

Roger-François Gauthier

\section{OpenEdition}

Édition électronique

URL : https://journals.openedition.org/ries/4563

DOI : $10.4000 /$ ries.4563

ISSN : 2261-4265

Éditeur

France Education international

Édition imprimée

Date de publication : 1 avril 2016

Pagination : 24-26

ISBN : 978-2-85420-610-4

ISSN : $1254-4590$

Référence électronique

Roger-François Gauthier, « Marie-France Lange et Nolwen Henaff (sous la direction de), "Internationalisation et transformation des systèmes éducatifs du Sud » », Revue internationale d'éducation de Sèvres [En ligne], 71 | avril 2016, mis en ligne le 01 avril 2016, consulté le 01 juillet 2021. URL : http://journals.openedition.org/ries/4563 ; DOI : https://doi.org/10.4000/ries.4563

Ce document a été généré automatiquement le 1 juillet 2021.

(c) Tous droits réservés 


\section{Marie-France Lange et Nolwen Henaff (sous la direction de), « Internationalisation et transformation des systèmes éducatifs du Sud»}

Revue Tiers-Monde, $\mathrm{n}^{\circ}$ 223, juil-sept. 2015

Roger-François Gauthier

1 Par définition collectifs, les numéros de revues peuvent selon les cas être lus soit dans la perspective mise en avant par les responsables du numéro, soit pour chacun des articles contributeurs. Cette livraison de Tiers-Monde (revue de l'Institut d'étude et de développement économique et social de l'Université Paris 1), qui consacre de temps à autre un numéro aux questions d'éducation, permet les deux entrées, les articles pouvant chacun susciter un intérêt autonome pour le lecteur, par leur méthode comme par leurs résultats.

2 L'intention générale du numéro, telle que le précise l'introduction de Marie-France Lange et Nolwen Henaff, est de se demander jusqu'à quel point il est pertinent, à propos de l'éducation, de mettre en dialogue les deux termes de mondialisation et d'internationalisation. Le premier évoque les tendances à la standardisation dans le cadre d'un futur système mondial de convergence, le second ce qui relève bien encore de l'action des États, mais dans un cadre qui passe peu à peu d'une logique de service public, encore tourné vers la formation des hommes, à une logique de prestations, de services divers, devenus concurrents, proposés eux-mêmes à d'autres pays. Les auteurs observent toutefois que les deux «modèles ", à peine distingués, se rejoignent, dans les évolutions contemporaines, la standardisation multipliant les opportunités pour les acteurs de passer d'un système à l'autre, et du coup en renforçant la mise en concurrence. 
3 Au cas où ce choix entre le quasi-même et le quasi-autre, entre le Charybde de la mondialisation et le Scylla de cette internationalisation laisserait encore au lecteur quelque illusion de l'intérêt de choisir, il est rappelé que tout se passe en tout état de cause dans un bain de recommandations coercitives, qui, dans un cadre mondialisé, installent un discours qui, autour de quelques mots tels que "autonomie", " compétences ", « décentralisation ", devient incontournable.

4 Il l'est d'autant plus que, dans les pays en développement, des bailleurs internationaux, de différents statuts qui n'ont en commun que de n'avoir aucune légitimité démocratique, l'imposent aux gouvernements en face de promesses d'aide.

5 L'intérêt de ce numéro par rapport à des choses déjà lues sur ces sujets est d'aller voir concrètement dans plusieurs systèmes éducatifs, et même pour certains articles, comme celui de Sarah Fichtner sur le Bénin, jusque dans les salles de classes, ce qu'il en est de l'inscription de ces idées voyageuses dans la réalité.

Les auteurs du numéro croient pouvoir observer trois mouvements :

- une «sédimentation» de ces réformes d'origine mondialisée quand elles impactent les réalités locales, cette sédimentation signifiant souvent une désémantisation de la réforme ;

- une paradoxale légitimation des réformes pour les responsables politiques de pays ayant perdu une part de leur souveraineté, en raison de leur renommée « internationale », ce dont Hélène Charton démonte de façon originale les mécanismes, en analysant la trajectoire biographique d'acteurs sénégalais entre leur pays et la scène internationale ;

- au bout du compte, une grave segmentation générale des systèmes, suite à divers mouvements d'importation partielle de modèles scolaires, dotés d'un sens qui n'était pas celui de la réforme ou ne couvrant qu'une partie d'un champ national désormais éclaté, comme cela est montré à propos de la formation professionnelle au Maroc, en Algérie et en Tunisie dans l'article de Saïd ben Sedrine et al.

7 Chacun de ces articles est tragique, pourrait-on dire, en ce qu'ils montrent tous les impasses où vont les pays, sans illusion, mais sans autre possibilité. Le conflit ivoirien, par exemple, étudié par Anne-Charlotte Triplet, quand à la destruction massive des écoles répondirent comme elles le purent des ONG qui, faisant le travail normal d'un gouvernement, risquaient de contribuer à sa perte de souveraineté. Même gouvernement qui, à la paix rendue tira pour partie sa légitimité de sa capacité à collecter des fonds internationaux qui le rendaient encore plus dépendant de l'aide.

De même, dans l'article de Sophie Lewandowski, la prétention bolivienne à une innovation curriculaire en rupture majeure, celle du "Vivir bien", construit politiquement contre le thème du "développement durable» dénoncé comme ne proposant pas vraiment de rupture vis-à-vis du développement capitaliste à faible préoccupation sociale et culturelle, n'apparaît pas comme une porte de sortie, à la fois par ses connotations religieuses et par finalement son hybridation avec des modèles éducatifs autres. De même, cette Université de Kinshasa, décrite par Marc Poncelet et al., qui se protège de l'internationalisation par un système d'éclatement du pouvoir en trente-six fiefs porteurs d'intérêts particuliers, ne montre pas de possibilité de solutions en dehors de celles qui sortent de la machine internationalisée.

9 L'article sur le Bénin déjà cité regarde comment la mise en œuvre de l'approche par compétences s'effectue, dans le triple cadre concret d'une classe, d'un module de formation des enseignants et d'une épreuve d'examen: demander de mettre en place un curriculum par compétences dans une école dont les principes autoritaires n'ont 
changé ni le visage du professeur ni celui d'un examen toujours tourné vers la régurgitation de savoirs, c'est créer un jeu de faux semblants (« teachers tend to copy the form and the structure of competency-based education, and pupils copy the content of what they are supposed to reproduce in tests $»)$.

Faux-semblant aussi mis en évidence dans l'article de Rachel Tsolomon Tsehaye qui s'intéresse, à partir de questionnement des acteurs, parents ou éducateurs, aux quatre systèmes éducatifs concurrents de Djibouti (en fait deux fois deux): on croit voir s'opposer deux conceptions de l'école, l'une et l'autre aidées par des pays étrangers, d'ailleurs, celle inspirée de l'Occident à prétention scientifique et universaliste, et celle inspirée de l'islam, qui s'oppose à la vision de la première, alors qu'en réalité s'opposent au sein de la société djiboutienne des milieux favorisés, à qui l'international ne fait pas peur (quitte à "panacher » pour leurs propres enfants) et des laissés pour compte de l'école comme de l'emploi, qui fréquentent medersas et école coraniques, et qui, n'ayant rien à perdre, peuvent « faire de la déviance, si ce n'est de la violence, une vertu».

11 Revue de chercheurs, cette livraison de Tiers-Monde peut décourager celui ou celle qui est investi(e) dans l'action: il ou elle gagnera toutefois à chercher les sens de ce qu'il fait dans ce paysage tellement truffé d'impasses.

\section{AUTEUR}

\section{ROGER-FRANÇOIS GAUTHIER}

Roger-François Gauthier, inspecteur général de l'administration de l'éducation nationale et de la recherche, est professeur associé à l'Université Paris-Descartes (France) et membre du Conseil supérieur des programmes. Ses travaux, au sein des institutions françaises et comme chercheur ou consultant auprès de plusieurs organisations internationales (Unesco, Organisation internationale de la Francophonie-OIF), relèvent soit d'études spécifiques à un pays soit des comparaisons internationales. Ils portent notamment sur les contenus d'enseignement et les politiques curriculaires. Courriel : roger-francois.gauthier@education.gouv.fr 